\title{
Sequencing and de novo assembly of a Dahlia hybrid cultivar transcriptome
}

\author{
Erik M. Lehnert ${ }^{1,2 *}$ and Virginia Walbot ${ }^{3}$ \\ 1 Department of Genetics, Stanford University School of Medicine, Stanford, CA, USA \\ 2 Department of Medical Microbiology and Immunology, University of Wisconsin School of Medicine, Madison, WI, USA \\ ${ }^{3}$ Department of Biology, Stanford University, Stanford, CA, USA
}

Edited by:

Gane Ka-Shu Wong, University of

Alberta, Canada

Reviewed by:

Xun Xu, BGI-Shenzhen, China

Jingfa Xiao, Chinese Academy of

Sciences, China

*Correspondence:

Erik M. Lehnert, Department of

Medical Microbiology and

Immunology, University of

Wisconsin School of Medicine,

1550 Linden Dr., Madison, WI

53706, USA

e-mail: lehnert@wisc.edu
Dahlia variabilis, with an exceptionally high diversity of floral forms and colors, is a popular flower amongst both commercial growers and hobbyists. Recently, some genetic controls of pigment patterns have been elucidated. These studies have been limited, however, by the lack of comprehensive transcriptomic resources for this species. Here we report the sequencing, assembly, and annotation of the transcriptome of the developing leaves, stems, and floral buds of $D$. variabilis. This resulted in 35,638 contigs, most of which seem to contain the complete coding sequence, and of which 20,881 could be successfully annotated by similarity to UniProt. Furthermore, we conducted a preliminary investigation to identify contigs with expression patterns consistent with tissue-specificity. These results will accelerate research into the genetic controls of pigmentation and floral form of $D$. variabilis.

Keywords: Dahlia, next-generation sequencing, de novo transcriptome assembly, anthocyanin biosynthesis, floral homeotic factors

\section{INTRODUCTION}

Horticultural dahlias (Dahlia variabilis) are among the most diverse in floral form and colors of all popular garden flowers. Dahlias are also an important contributor to the more than US $\$ 100$ Billion worldwide flower and potted plant market. Taxonomically dahlias are Compositae, and the center of species diversity is in Mexico and Central America. Likely through crossbreeding of two wild species followed by human selection since 1800 (Gatt et al., 1998), thousands of varieties have been generated by European horticulturists who prized novelty in overall flower size, color, petal number, and petal form.

Garden dahlias are proposed to be recent octoploid derivatives $(2 \mathrm{~N}=64)$ (Gatt et al., 1998) from crosses between two natural species. Although this high ploidy may have been expected to result in redundancy of genetic factors, loci implicated in flower color have been elucidated and generally behave as diploid factors (Bate-Smith et al., 1955). More recently, genes that are highly expressed in flowers and encoding anthocyanin pathway enzymes have been cloned (Suzuki et al., 2002; Ohno et al., 2011b, 2013) and some enzymes characterized (Yamaguchi et al., 1999; Ogata et al., 2001). Furthermore, a partial reference transcriptome assembly was performed for a comparative genomics study of Compositae, but the majority of contigs did not contain the complete coding sequence (Hodgins et al., 2014).

Given the relatively low cost of deep transcriptome analysis using next-generation DNA sequencing, we wished to determine if reasonable descriptions of messenger RNA diversity could be obtained for leaves, stems, and floral buds of dahlia. The contigs assembled from the transcriptome data are sufficient to propose more than 20,000 likely protein coding genes in this species and can thus serve as a standard for allele comparison among dahlia varieties and for future studies of transposon-mediated variegation in anthocyanin pigmentation and the control of floral form. A cultivar containing anthocyanin in the stems and leaves and flavonoid precursors and anthocyanin in the petals was selected to assess recovery of known pigment factor transcripts as a test of the transcriptome completeness.

\section{MATERIALS AND METHODS PLANT GROWTH AND RNA ISOLATION}

The cultivar "Rio Riata," originally obtained from Corralitos Gardens (http://www.cgdahlias.com/), was grown outdoors at Stanford University; this variety has 8 orange-red petals with yellow-tips, purple (anthocyanin pigment) stems, and green leaves tinged with purple (http://www.stanford.edu/group/ dahlia_genetics/cultivars/rio_riata/rio_riata.htm). Total RNA was extracted from newly emerged stems, leaves, and buds $(\sim 0.2-$ $1 \mathrm{~cm}$ in diameter) using the RNAqueous-4PCR Kit (Ambion AM1914, Grand Island NY) following the manufacturer's instructions. The RNA-integrity number (RIN) of each sample was determined using an Agilent 2100 Bioanalyzer, Santa Clara CA), and only samples with a RIN $\geq 8$ were used. Approximately $1 \mu \mathrm{g}$ of total RNA was processed (including a poly- $\mathrm{A}^{+}$-selection step) from the leaf, stem, and bud extracts using the TruSeq RNA Sample Prep Kit (Illumina FC-122-1001, Hayward CA) following the manufacturer's instructions to produce indexed libraries. The resulting libraries were pooled based on their indices (as described in the kit instructions). Clustering and sequencing were performed by the Stanford Center for Genomics and Personalized Medicine using an Illumina HiSeq 2000 sequencer to generate 101-bp paired-end reads. Accession numbers for reads from bud, leaf, and stem are: SRR1222985, SRR1226613, SRR1226614, respectively. 


\section{READ FILTERING, TRANSCRIPTOME ASSEMBLY, AND ANNOTATION}

Transcriptome assembly was performed combining reads from all three tissues. Prior to assembly, the reads were processed as previously described (Lehnert et al., 2014) and outlined here: (1) the first 6 bp from the $5^{\prime}$ end of each read was discarded; (2) reads of $<60$ bp or containing $\geq 1 \mathrm{~N}$ were discarded; (3) low quality reads were discarded (if $>10$ of the first 35 bases had quality scores $<30$ ); and (4) reads were trimmed to the first position for which a sliding 4-bp window had an average quality-score of $<20$. The remaining read-pairs were then processed using FLASH (Magoc and Salzberg, 2011) to join reads whose ends overlapped by $\geq 10$ bp with no mismatches. Finally, adapter sequences were removed using cutadapt (Martin, 2011) with default settings.

The processed reads were assembled using a 43-bp $k$-mer with the Velvet/Oases assembler (Velvet version 1.2.09 and Oases version 0.2.08) (Zerbino and Birney, 2008; Schulz et al., 2012) with the default settings. To choose a single representative contig from each "locus" (the Oases term for a connected component in the de Bruijn graph, which presumably consists of alternative transcripts, alleles, and extremely similar paralogs), the script “process_oases_transcripts.py" was used (Yang and Smith, 2013). This script designates as representative the contig with the highest geometric mean $k$-mer coverage that is also at least $30 \%$ as long as the longest contig within the locus. Only contigs longer than $300 \mathrm{bp}$ were retained in the resulting set of representative contigs. Contigs were post-processed to remove terminal Ns, and such that internal runs of Ns were fewer than 14 bases in length.

To assign putative functional roles to the representative contigs, they were aligned to the SwissProt protein database and the NCBI Non-Redundant Protein Database (nr) using the blastx program from the standalone BLAST 2.2.25+software suite (Camacho et al., 2009) with an E-value cutoff of 1e-5. The Blast2GO software package (Conesa et al., 2005) was used with default settings to assign Gene Ontology (GO) terms and Enzyme codes to the predicted proteins based on their alignments to SwissProt. Blast2GO was also used to identify protein domains by InterProScan (Quevillon et al., 2005), whose associated GO terms were merged with those identified by alignment to SwissProt.

\section{EXPRESSION ANALYSIS}

The trimmed forward reads were aligned to the representative contigs using bwa (Li and Durbin, 2009). Aligned reads were counted using the samtools (Li et al., 2009; minimum mapping quality 30). The R package DESeq2 (Anders and Huber, 2010) was used to normalize read counts by library size.

\section{RESULTS}

\section{SEOUENCING AND DE NOVO TRANSCRIPTOME ASSEMBLY}

A set of 149,304,876 reads of 101-bp was generated in the initial sequencing run. After trimming and discarding low-quality reads, $\sim 73$ million reads from buds, leaves, and stems (see Table 1) were assembled into 53,037 loci containing 122,053 contigs. After removing contigs that fell below the 300-bp length cutoff, choosing representative contigs for each locus, and removing unnecessary Ns (see Materials and Methods), 35,638 representative contigs remained. These ranged in size from $269 \mathrm{bp}$ to 13,886 (see Table 2). These contigs have been deposited in the NCBI
TSA (accession \# GBDN01000001-GBDN01035638) and can also be found at (http://www.stanford.edu/group/dahlia_genetics/). $20,881(58.5 \%)$ of contigs could be annotated by BLAST alignment to the UniProt protein database. Using these alignments and the results of InterProScan protein motif searches, 122,654 GO terms were assigned to $21,576(60.5 \%)$ of the contigs (see Table 3).

\section{COMPARISON TO KNOWN COMPONENTS OF ANTHOCYANIN BIOSYNTHESIS IN DAHLIAS AND IDENTIFICATION OF ADDITIONAL GENE COPIES}

Previous research has identified and sequenced several genes of the dahlia anthocyanin biosynthetic pathway (Suzuki et al., 2002;

Table 1 | Summary of read metrics by tissue of origin.

\begin{tabular}{lccc}
\hline & $\begin{array}{c}\text { Total number of } \\
\text { reads }\end{array}$ & $\begin{array}{c}\text { Total nucleotides } \\
\text { (Mb) }\end{array}$ & $\begin{array}{c}\text { Average Length } \\
\text { (bp) }\end{array}$ \\
\hline $\begin{array}{l}\text { Floral } \\
\text { (paired) }\end{array}$ & $12,792,456$ & 1118 & 87 \\
$\begin{array}{l}\text { Floral (flash } \\
\text { extended }\end{array}$ & $9,456,389$ & 1264 & 133 \\
$\begin{array}{l}\text { Leaf (paired) } \\
\text { Leaf (flash } \\
\text { extended) }\end{array}$ & $14,541,486$ & 1258 & 133 \\
$\begin{array}{l}\text { Stem } \\
\text { (paired) }\end{array}$ & $13,742,631$ & 1840 & 86 \\
$\begin{array}{l}\text { Stem (flash } \\
\text { extended) }\end{array}$ & $11,455,453$ & 958 & 130 \\
Total & $73,040,117$ & 1499 & - \\
\hline
\end{tabular}

a Flash extended reads are paired reads that had 10-bp or more of perfect overlap at the ends of their sequence. These were joined to make longer reads, and are not included in the counts of paired reads (see Materials and Methods).

Table 2 | Size distribution of the representative contigs.

\begin{tabular}{lcc}
\hline & Representative contigs & All contigs \\
\hline Number of loci & 35,638 & 53,037 \\
Number of contigs & 35,638 & 122,053 \\
Median contig size (bp) & 906 & 943 \\
Mean contig size (bp) & 1166 & 1209 \\
Minimum contig size (bp) & 269 & 100 \\
Maximum contig size (bp) & 13,886 & 17,090 \\
Total bases in assembly (Mb) & 41.6 & 148 \\
\hline
\end{tabular}

Table 3 | Summary of alignments to SwissProt and $\mathbf{n r}$.

\begin{tabular}{lcccc}
\hline $\begin{array}{l}\text { Number of } \\
\text { contigs }\end{array}$ & $\begin{array}{c}\text { Number (\%) of } \\
\text { contigs } \\
\text { aligned to } \\
\text { SwissProt }\end{array}$ & $\begin{array}{c}\text { Number } \\
\left(\%^{\mathbf{b}}\right) \text { of } \\
\text { unique } \\
\text { accessions }\end{array}$ & $\begin{array}{c}\text { Number (\%) of } \\
\text { contigs } \\
\text { aligned to } \mathbf{n r}^{\mathbf{a}}\end{array}$ & $\begin{array}{c}\text { Number } \\
(\% \mathbf{\%}) \text { of } \\
\text { unique } \\
\text { accessions }\end{array}$ \\
\hline 35,638 & $20,880(58.6)$ & $10,603(50.8)$ & $26,940(75.6)$ & $21,376(79.3)$
\end{tabular}

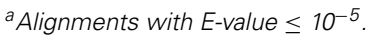

${ }^{b}$ As \% of all alignments. 
Ohno et al., 2011a). To investigate assembly quality, we compared these previously sequenced genes to the contigs in our assembly. As shown in Table 4, we identified all the genes previously known to be involved in the synthesis of the anthocyanidins beginning with chalcone synthase. Furthermore, we identified additional copies for several pathway genes by a best-reciprocalblast approach, thereby expanding the dahlia gene list for the phenylpropanoid pathways. For example, we identified a total of six putative chalcone synthase genes, four more than were previously known. All contigs identified contained the complete coding sequence of the enzyme (as determined by alignment of sequence to the stop and start codon of reference protein), except for one chalcone synthase and for DvIVS, a basic helix-loop-helix transcription factor required for anthocyanin synthesis, in which the cDNA was split between two contigs.

\section{INVESTIGATING TISSUE-SPECIFIC EXPRESSION}

To achieve statistical power in assigning contigs to classes differentially expressed between tissue types, biological replicates would be required. Nonetheless, as a preliminary investigation that might guide future experimental design, we identified contigs with expression patterns consistent with tissue specificity. To do this, we used DESeq to perform a regularized log2 transformation on the read counts; this analysis generates normalized

\begin{tabular}{|c|c|c|c|}
\hline $\begin{array}{l}\text { Putative } \\
\text { enzyme }\end{array}$ & $\begin{array}{c}\text { Locus \# / } \\
\text { transcript \# }\end{array}$ & $\begin{array}{c}\text { Tissue- } \\
\text { specificity }^{a}\end{array}$ & $\begin{array}{l}\text { Previously } \\
\text { identified? }\end{array}$ \\
\hline $\mathrm{CHS}$ & $27 / 7$ & All & $\mathrm{CHS} 2^{\mathrm{b}}$ \\
\hline $\mathrm{CHS}$ & $49578 / 1$ & Elevated in stem & $\mathrm{CHS} 1^{\mathrm{b}}$ \\
\hline $\mathrm{CHS}$ & $7505 / 1$ & Decreased in leaf & \\
\hline $\mathrm{CHS}$ & $3129 / 1$ & All & \\
\hline $\mathrm{CHS}$ & $18623 / 1$ & Elevated in bud & \\
\hline $\mathrm{CHS}$ & $9318 / 2^{c}$ & Elevated in bud & \\
\hline $\mathrm{CHI}$ & $977 / 3$ & All & $\mathrm{CHI}$ \\
\hline $\mathrm{CHI}$ & $2352 / 8$ & All & \\
\hline $\mathrm{CHI}$ & $13838 / 4$ & All & \\
\hline DvIVS & $7738 / 1 ; 36303 / 2^{d}$ & All & DvIVSc \\
\hline FLS & $4250 / 4$ & Elevated in stem & \\
\hline $\mathrm{F}^{\prime} \mathrm{H}$ & $824 / 8$ & All & \\
\hline $\mathrm{F}^{\prime} \mathrm{H}$ & $16120 / 4$ & All & \\
\hline F3'H & $18455 / 5$ & All & \\
\hline DFR & $44537 / 3$ & All & $\mathrm{DFR}^{\mathrm{b}}$ \\
\hline DFR & $23328 / 5$ & Decreased in bud & \\
\hline DFR & $11673 / 3$ & All & \\
\hline DFR & $27896 / 1$ & Decreased in bud & \\
\hline ANS & $45613 / 2$ & All & $\mathrm{ANS}^{\mathrm{b}}$ \\
\hline ANS & $27069 / 1$ & All & \\
\hline $\mathrm{F} 3 \mathrm{H}$ & $22534 / 2$ & All & $\mathrm{F} 3 \mathrm{H}^{\mathrm{b}}$ \\
\hline $\mathrm{F} 3 \mathrm{H}$ & $33298 / 1$ & All & \\
\hline $\mathrm{F} 3 \mathrm{H}$ & $39959 / 2$ & Decreased in bud & \\
\hline $\mathrm{F} 3 \mathrm{H}$ & $43247 / 1$ & All & \\
\hline
\end{tabular}

axpressed in all three samples at levels that did not differ enough to be designated as consistent with tissue-specific expression, designated as All. b Ohno et al., 2011b. ' Ohno et al., 2011a. ${ }^{d}$ These loci lacked either conserved start or stop codons. expression values after accounting for the differences in sequencing depth. We assigned a tentative tissue source to a contig by the following procedures: (1) We calculated the standard deviation of expression values across all tissues; (2) we calculated the standard deviation of expression values for each pair of tissues; (3) a contig's expression was classified as elevated or decreased in a specific tissue if the standard deviation of expression values of the pair of other tissues was 3-fold less than the standard deviation of the expression values of all three tissues and the standard deviation of the three tissues expression value was great than $10 \%$ the mean expression value. This selected for contigs that showed stable expression in two of three tissues, with a large difference in read counts in the third. Table 4 presents this analysis for the anthocyanin biosynthetic pathway, and Table $\mathbf{5}$ considers all classes of representative contigs.

This analysis resulted in tentative tissue-specific expression patterns for 8823 contigs (see Table 5). Given the distribution of pigment in Rio Riata, genes essential for anthocyanin synthesis would be expected in the transcriptomes of all three organs, and they were found. Interestingly, while genes for some of the enzymes exhibited similar expression in all three tissues, and all tissues had all the essential components of the pathway for pigment synthesis, some copies (alleles) exhibited patterns consistent with tissue-specific expression (see Table 4). We verified that these results were not affected by reads mapping to multiple contigs, as using only uniquely mapping reads gave nearly identical results. This implies that the apparent diploid nature of dahlia floral pigmentation genes may reflect not only the loss of function of redundant gene-copies, but also evolved tissue-specific expression patterns for some of the redundant genes, a process termed subfunctionalization. As a second test of the classification approach, we investigated the tissue-specificity of contigs annotated as floral homeotic proteins. Of the 22 contigs annotated as similar to floral homeotic proteins, 12 were elevated in buds as expected, while the remainder were classified as lacking tissue specificity (see Table 6).

\section{DISCUSSION}

Despite the large genome size of $D$. variabilis (9.62 pg; Temsch et al., 2008) and the expectation of two to four paralogs per locus type in an octoploid (Gatt et al., 1998), a paired-end read strategy using next generation sequencing yielded sufficient data to assemble more than 20,000 contigs that could be annotated with GO terms. As flowering plants sequenced to date contain $25,000-40,000$ genes (e.g., Arabidopsis Genome Initiative, 2000; Matsumoto et al., 2005; Amborella Genome Project, 2013) it is likely that the transcriptome data define approximately half

Table 5 | Tentative tissue specificity of contigs.

\begin{tabular}{lc}
\hline Expression pattern & Number in this class \\
\hline Decreased in buds & 1407 \\
Elevated in buds & 390 \\
Decreased in leaves & 2059 \\
Elevated in leaves & 1409 \\
Decreased in stems & 2690 \\
Elevated in stems & 868
\end{tabular}


Table 6 | Putative floral homeotic genes in Dahlia.

\begin{tabular}{|c|c|c|c|c|c|c|c|}
\hline $\begin{array}{l}\text { Locus\# / } \\
\text { transcript\# }\end{array}$ & $\begin{array}{l}\text { Match to floral } \\
\text { homeotic gene }\end{array}$ & $\begin{array}{c}\text { Best blast hit } \\
\text { accession number }\end{array}$ & $e$-Value & $\begin{array}{l}\text { Read counts } \\
\text { in bud }\end{array}$ & $\begin{array}{l}\text { Read counts } \\
\text { in leaf }\end{array}$ & $\begin{array}{l}\text { Read counts } \\
\text { in stem }\end{array}$ & $\begin{array}{l}\text { Tissue- } \\
\text { specificity }\end{array}$ \\
\hline $312 / 8$ & PMADS2 & Q07474 & $4.47 e-71$ & 5697 & 1 & 69 & Elevated in bud \\
\hline $3477 / 2$ & APETALA1 & Q41276 & $9.28 e-92$ & 2034 & 0 & 22 & Elevated in bud \\
\hline $16671 / 2$ & AGAMOUS & Q03489 & $8.38 e-119$ & 1192 & 3 & 17 & Elevated in bud \\
\hline $324 / 3$ & AGAMOUS & Q40872 & $3.30 \mathrm{e}-128$ & 1163 & 7 & 14 & Elevated in bud \\
\hline $5269 / 3$ & DEFICIENS & P23706 & $3.68 e-96$ & 895 & 0 & 18 & Elevated in bud \\
\hline $5867 / 6$ & DEFICIENS & P23706 & $1.82 e-91$ & 729 & 2 & 18 & Elevated in bud \\
\hline $3943 / 2$ & AGAMOUS & Q03489 & $9.65 e-133$ & 645 & 41 & 11 & Elevated in bud \\
\hline $643 / 1$ & AGAMOUS & Q40872 & $5.90 e-127$ & 637 & 3 & 13 & Elevated in bud \\
\hline 8582/1 & AGAMOUS & Q40872 & $4.94 e-122$ & 245 & 0 & 5 & Elevated in bud \\
\hline $4783 / 1$ & AGAMOUS & Q01540 & $1.72 \mathrm{e}-06$ & 157 & 0 & 0 & Elevated in bud \\
\hline $9368 / 1$ & PMADS1 & Q07472 & $1.28 e-69$ & 67 & 6 & 1 & Elevated in bud \\
\hline $9276 / 1$ & APETALA2 & P47927 & $1.35 e-36$ & 33 & 0 & 3 & Elevated in bud \\
\hline $7009 / 1$ & DEFICIENS & P23706 & $2.93 e-94$ & 1675 & 27 & 227 & All \\
\hline $1095 / 3$ & AGAMOUS & Q40872 & $4.44 \mathrm{e}-137$ & 1118 & 0 & 54 & All \\
\hline $15403 / 1$ & APETALA2 & P47927 & $8.53 e-111$ & 693 & 1197 & 616 & All \\
\hline $22399 / 7$ & APETALA2 & P47927 & $5.50 e-90$ & 350 & 557 & 323 & All \\
\hline $17401 / 4$ & APETALA2 & P47927 & $1.45 e-85$ & 214 & 240 & 502 & All \\
\hline $23847 / 1$ & APETALA2 & P47927 & $3.84 \mathrm{e}-88$ & 140 & 8 & 61 & All \\
\hline $40009 / 3$ & APETALA2 & P47927 & $7.44 \mathrm{e}-101$ & 135 & 105 & 347 & All \\
\hline $15550 / 2$ & APETALA2 & P47927 & $2.8 e-76$ & 98 & 19 & 40 & All \\
\hline $31911 / 1$ & APETALA1 & D7KWY6 & $1.06 e-09$ & 30 & 14 & 6 & All \\
\hline 38896/1 & APETALA2 & P47927 & $3.57 e-106$ & 16 & 3 & 183 & All \\
\hline
\end{tabular}

${ }^{a}$ Expressed in all three samples at levels that did not differ enough to be designated as consistent with tissue-specific expression, designated as All.

of dahlia loci. Genes expressed in seeds, roots, and non-abundant specialized cell types were likely missed as well as genes expressed during biotic or abiotic stress. Given the scanty knowledge about Dahlia genes, this new transcriptome resource can serve as a guide in gene identification and cloning, as a standard for comparison among varieties and species, and as a database for identifying genes unique to Dahlia and paralogs and orthologs shared with other composites, flowering plants, and all plants. Furthermore, we identified contigs whose expression patterns were consistent with tissue-specificity; future researchers may test our assignments by qPCR or further sequencing. Our method was limited by a lack of biological replicates, which prevents any estimate of the variability of expression of genes within a tissue-type. For this reason, we attempted to be conservative in our calling of specificity and have likely both failed to identify some contigs whose expression is highly enriched in certain tissues, as well as potentially misassigned contigs whose expression is not tissue-specific but is highly variable. However, we believe this resource will be of use for researchers attempting to clone cDNAs or perform in situ hybridization to localize specific transcripts. This report indicates that reference transcriptomes for plant species with complex genomes are a feasible and relatively inexpensive method for generating a toolkit for molecular and genetic analysis.

\section{AUTHOR CONTRIBUTIONS}

Erik M. Lehnert performed all of the experiments; Virginia Walbot maintained the dahlias; both authors contributed to design of data collection and analysis and manuscript writing and editing.

\section{ACKNOWLEDGMENTS}

We thank Tim Culbertson and Blaine Marchant for constructing the Stanford Dahlia Project website (http://web.stanford.edu/ group/dahliagenetics/dahliareferencetranscriptome.htm) and for starting the dahlia collection. John Fernandes incorporated the transcriptome data into the website. This effort was supported by the Savitsky Fund and by the outreach component of a National Science Foundation grant (PGRP 07-01880) to Virginia Walbot. Erik M. Lehnert was supported by the Gordon and Betty Moore Foundation (grant \#2629).

\section{SUPPLEMENTARY MATERIAL}

The Supplementary Material for this article can be found online at: http://www.frontiersin.org/journal/10.3389/fpls.2014.00340/ abstract

\section{REFERENCES}

Amborella Genome Project. (2013). The Amborella genome and the evolution of flowering plants. Science 342, 1241089. doi: 10.1126/science. 1241089

Anders, S., and Huber, W. (2010). Differential expression analysis for sequence count data. Genome Biol. 11, R106. doi: 10.1186/gb-2010-11-10-r106

Arabidopsis Genome Initiative. (2000). Analysis of the genome sequence of the flowering plant Arabidopsis thaliana. Nature 408, 796-815. doi: $10.1038 / 35048692$

Bate-Smith, E. C., Swain, T., and Nördstrom, C. G. (1955). Chemistry and inheritance of flower colour in the Dahlia. Nature 176, 1016-1018. doi: $10.1038 / 1761016 \mathrm{a} 0$

Camacho, C., Coulouris, G., Avagyan, V., Ma, N., Papadopoulos, J., Bealer, K., et al. (2009). BLAST+: architecture and applications. BMC Bioinformatics 10:421. doi: 10.1186/1471-2105-10-421 
Conesa, A., Götz, S., García-Gómez, J., Terol, J., Talón, M., and Robles, M. (2005). Blast2GO: a universal tool for annotation, visualization and analysis in functional genomics research. Bioinformatics 21, 3674-3676. doi: 10.1093/bioinformatics/bti610

Gatt, M., Ding, H., Hammet, K., and Murray, B. (1998). Polyploidy and evolution in wild and cultivated Dahlia species. Ann. Bot. 81, 647-656. doi: 10.1006/anbo.1998.0614

Hodgins, K. A., Lai, Z., Oliveira, L. O., Still, D. W., Scascitelli, M., Barker, M. S., et al. (2014). Genomics of Compositae crops: reference transcriptome assemblies and evidence of hybridization with wild relatives. Mol. Ecol. Resour. 14, 166-1177. doi: 10.1111/1755-0998.12163

Lehnert, E. M., Mouchka, M. E., Burriesci, M. S., Gallo, N. D., Schwarz, J. A., and Pringle, J. R. (2014). Extensive differences in gene expression between symbiotic and aposymbiotic cnidarians. G3 4, 277-295. doi: 10.1534/g3.113. 009084

Li, H., and Durbin, R. (2009). Fast and accurate short read alignment with Burrows-Wheeler transform. Bioinformatics 25, 1754-1760. doi: 10.1093/bioinformatics/btp324

Li, H., Handsaker, B., Wysoker, A., Fennell, T., Ruan, J., Homer, N., et al. (2009). The sequence alignment/map format and SAMtools. Bioinformatics 25, 2078-2079. doi: 10.1093/bioinformatics/btp352

Magoc, T., and Salzberg, S. L. (2011). FLASH: fast length adjustment of short reads to improve genome assemblies. Bioinformatics 27, 1-8. doi: 10.1093/bioinformatics/btr507

Martin, M. (2011). Cutadapt removes adapter sequences from highthroughput sequencing reads. EMBnet.J. 17, 10-12. doi: 10.14806/ej. 17.1.200

Matsumoto, T., Wu, J. Z., Kanamori, H., Katayose, Y., Fujisawa, M., Namiki, N., et al. (2005). The map-based sequence of the rice genome. Nature 436, 793-800. doi: 10.1038/Nature03895

Ogata, J., Sakamoto, T., Yamaguchi, M., Kawanobu, S., and Yoshitama, K. (2001). Isolation and characterization of anthocyanin 5-O-glucosyltransferase from flowers of Dahlia variabilis. J. Plant Physiol. 158, 709-714. doi: 10.1078/01761617-00370

Ohno, S., Deguchi, A., Hosokawa, M., Tatsuzawa, F., and Doi, M. (2013). A basic helix-loop-helix transcription factor DvIVS determines flower color intensity in cyanic dahlia cultivars. Planta 238, 331-343. doi: 10.1007/s00425-0131897-x

Ohno, S., Hosokawa, M., Hoshino, A., Kitamura, Y., Morita, Y., Park, K.-I., et al. (2011a). A bHLH transcription factor, DvIVS, is involved in regulation of anthocyanin synthesis in dahlia (Dahlia variabilis). J. Exp. Bot. 62, 5105-5116. doi: 10.1093/jxb/err216

Ohno, S., Hosokawa, M., Kojima, M., Kitamura, Y., Hoshino, A., Tatsuzawa, F., et al. (2011b). Simultaneous post-transcriptional gene silencing of two different chalcone synthase genes resulting in pure white flowers in the octoploid dahlia. Planta 234, 945-958. doi: 10.1007/s00425-011-1456-2

Quevillon, E., Silventoinen, V., Pillai, S., Harte, N., Mulder, N., Apweiler, R., et al. (2005). InterProScan: protein domains identifier. Nucl. Acids Res. 33, W116-W120. doi: 10.1093/nar/gki442

Schulz, M. H., Zerbino, D. R., Vingron, M., and Birney, E. (2012). Oases: robust de novo RNA-seq assembly across the dynamic range of expression levels. Bioinformatics 28, 1086-1092. doi: 10.1093/bioinformatics/bts094

Suzuki, H., Nakayama, T., Yonekura-Sakakibara, K., Fukui, Y., Nakamura, N., Yamaguchi, M., et al. (2002). cDNA cloning, heterologous expressions, and functional characterization of malonyl-coenzyme a: anthocyanidin 3o-glucoside-6"-o-malonyltransferase from dahlia flowers. Plant Physiol. 130, 2142-2151. doi: 10.1104/pp.010447

Temsch, E. M., Greilhuber, J., and Hammett, K. R. W. (2008). Genome size in Dahlia Cav. (Asteraceae-Coreopsideae). Plant Syst. Evol. 276, 157-166. doi: 10.1007/s00606-008-0077-0

Yamaguchi, M. A., Oshida, N., Nakayama, M., Koshioka, M., Yamaguchi, Y., and Ino, I. (1999). Anthocyanidin 3-glucoside malonyltransferase from Dahlia variabilis. Phytochemistry 52, 15-18. doi: 10.1016/S0031-9422(99) 00099-0

Yang, Y., and Smith, S. A. (2013). Optimizing de novo assembly of short-read RNAseq data for phylogenomics. BMC Genomics 14:328. doi: 10.1186/1471-216414-328

Zerbino, D. R., and Birney, E. (2008). Velvet: algorithms for de novo short read assembly using de Bruijn graphs. Genome Res. 18, 821-829. doi: 10.1101/gr.074492.107

Conflict of Interest Statement: The authors declare that the research was conducted in the absence of any commercial or financial relationships that could be construed as a potential conflict of interest.

Received: 22 May 2014; paper pending published: 06 June 2014; accepted: 25 June 2014; published online: 17 July 2014.

Citation: Lehnert EM and Walbot $V$ (2014) Sequencing and de novo assembly of a Dahlia hybrid cultivar transcriptome. Front. Plant Sci. 5:340. doi: 10.3389/fpls. 2014.00340

This article was submitted to Plant Genetics and Genomics, a section of the journal Frontiers in Plant Science.

Copyright (C) 2014 Lehnert and Walbot. This is an open-access article distributed under the terms of the Creative Commons Attribution License (CC BY). The use, distribution or reproduction in other forums is permitted, provided the original author(s) or licensor are credited and that the original publication in this journal is cited, in accordance with accepted academic practice. No use, distribution or reproduction is permitted which does not comply with these terms. 